\title{
El papel de la Unión Europea en la gobernanza global en materia climática
}

\author{
Dra. Lara Lázaro Touza \\ Profesora del Instituto de Empresa e Investigadora del Real Instituto Elcano
}

\begin{abstract}
Sumario: I. El problema, las soluciones y los procesos internacionales. - II. Proceso, causas, causantes y consecuencias. - III. La gobernanza global del cambio climático y la UE 1. La política climática de la UE. 2. ¿Qué se ha conseguido hasta la fecha? - IV. Retos pendientes. - V. Conclusiones. - VI. Bibliografía
\end{abstract}

Resumen: La Unión Europea (UE) ha buscado de manera proactiva el liderazgo en la gobernanza global del cambio climático desde finales de los años 80 del siglo XX. Esto ha sido así a pesar de una demanda asimétrica de acción climática por parte de los Estados miembros y de la complejidad que conlleva la acción cooperativa internacional. Los hechos muestran un liderazgo direccional por parte de la UE en determinados momentos y en algunos aspectos de las negociaciones climáticas. No obstante, tanto factores internos como externos, incluyendo el peso económico y político de otros grandes contaminadores, han relegado a la UE a un segundo plano, por ejemplo en la $\mathrm{CdP} 15^{1}$. El objetivo de este artículo es analizar el papel de la UE en la gobernanza global del cambio climático, los factores que influyen en el papel de la UE como líder direccional y los retos pendientes en materia de gobernanza climática.

Palabras clave: Cambio climático, gobernanza, Unión Europea, política climática.

Abstract: The European Union (EU) has proactively sought to become a leader in the global governance of climate change since the late 80's of the $X X^{\text {th }}$ century. This has been so despite an asymmetric demand for climate action on the part of Member States and despite the complexity of cooperative international action. Past events are indicative of the existence of directional leadership by the EU at certain points in time and in certain aspects of climate negotiations. However, both internal factors and external factors, such as the economic and political clout of other large polluters, have sidelined the EU for example during COP152. The objective of this article is to analyse the role of the EU in the global governance of climate change, the variables that have a bearing on the role of the EU as a directional leader and the pending challenges in the governance of climate change.

Keywords: Climate Change, governance, European Union, climate policy.

${ }^{1}$ CdP es el acrónimo de Conferencia de las Partes de la Convención Marco de las Naciones Unidas sobre el Cambio Climático (CMNUCC).

${ }^{2} \mathrm{COP}$ is the acronym for the United Nations Framework Convention on Climate Change (UNFCCC). 


\section{El problema, las soluciones y los procesos internacionales}

La Agencia Europea del Medio Ambiente nos dice que el clima de la tierra depende de varios factores. Estos factores son: la energía que recibe la tierra del sol, la superficie terrestre, los aerosoles y los gases de efecto invernadero (GEI). Cambios en estas variables transforman los patrones climáticos. El panel intergubernamental de expertos sobre cambio climático, que recopila y consensúa desde 1988 el conocimiento científico en materia climática, define el término cambio climático como aquellas fluctuaciones en la media o en la variabilidad de los patrones climáticos (temperaturas, precipitaciones y viento) a largo plazo, es decir, tres décadas o más. El origen de dichos cambios en las variables que determinan el clima puede ser tanto natural como antropogénico ${ }^{3}$. Sin embargo, hay pocas dudas en la literatura científica verificada por pares sobre la imposibilidad de explicar las concentraciones actuales de gases de efecto invernadero en la atmósfera, sin tener en cuenta las emisiones derivadas de la quema de combustibles fósiles que ha tenido lugar desde la revolución industrial.

Desde una perspectiva económica, la existencia de un clima estable, que permite la vida en la tierra y el desarrollo de toda actividad económica y social, es un bien público global cuyas características básicas son: la no rivalidad y la no exclusión. La no rivalidad implica que el uso y disfrute de un clima estable por parte de un individuo no disminuye la capacidad en el uso y disfrute de un clima estable por parte de otro individuo. La no exclusión se refiere a que, una vez que tenemos un clima estable, es muy difícil (o imposible) evitar el disfrute de dicho clima a ningún individuo. El problema radica en que la existencia de bienes públicos constituye un fallo de mercado. Este fallo de mercado conlleva una asignación subóptima de los recursos en la que hay un nivel de emisión de gases de efecto invernadero ineficiente.

Desde el punto de vista de la política, el cambio climático se conoce como el «problema malvado» (the wicked problem) ${ }^{4}$ con múltiples facetas. En primer lugar, se trata de un problema que requiere una transformación profunda del modelo económico, productivo y de consumo que desafía los valores e instituciones existentes. En este sentido es interesante la reflexión de Erik Assadourian ${ }^{5}$ sobre la necesidad de un cambio cultural para asegurar un clima estable. Assadourian aboga por una transformación desde la

${ }^{3}$ Intergovernmental Panel on Climate Change (IPCC), Fact sheet: Climate change science - the status of climate change science today, 2011.

${ }^{4}$ JORDAN, A., et al., Climate Change Policy in the European Union. Confronting the Dilemmas of Mitigation and Adaptation, Cambridge, Cambridge University Press, 2010.

5 ASSADOURIAN, E., «Cultural Change for a Bearable Climate», Real Instituto Elcano, ARI 163/2010-17/11/2010, 2010. 
cultura del consumismo hacia la cultura de la sostenibilidad ${ }^{6}$ a través de un cambio en la demanda de productos y servicios que resulte en la elección de productos y servicios más respetuosos con el entorno (choice editing).

Un problema añadido, desde el prisma del decisor político, es el hecho de que el cambio climático se ha visto tradicionalmente como un problema complejo en el que la interacción entre ciencia y política no está perfectamente coordinada. En relación a esta interacción, la reflexión de Tomas Koetz, et al. ${ }^{7}$ sobre la necesidad de construir puentes de interlocución entre la ciencia y la política para mejorar la gobernanza ambiental global es de gran interés en la solución de problemas como el cambio climático.

Además, al igual que ocurre con muchos otros problemas ambientales, el ciudadano se muestra ambivalente hacia el problema del cambio climático. Por un lado, declaramos estar preocupados por el cambio climático, pero por otro no queremos cambiar nuestro estilo de vida y de consumo para solucionar el problema. El reto pendiente en este sentido es aumentar la información sobre el problema y sus soluciones a la vez que aumentamos la coherencia entre nuestras intenciones y nuestras acciones ${ }^{8}$.

Un factor adicional que dificulta las acciones para reducir las emisiones presentes de GEI es el hecho de que hay un lapso de tiempo relativamente largo entre las emisiones de gases de efecto invernadero y las consecuencias derivadas de dichas emisiones. El hecho de que, en general, tendamos a descontar las acciones futuras (dándole más importancia a los costes y beneficios presentes que a los costes y beneficios futuros) hace que sea complejo motivar a los estados, gobernados a su vez por ciclos políticos a corto plazo, y a los individuos.

Finalmente, el hecho de tener que gestionar un problema con multitud de actores que tienen a su vez distintos niveles de desarrollo, prioridades diversas, niveles de exposición al cambio climático desiguales, diferentes capacidades de adaptación, con poder asimétrico y, además tener que involucrar a la práctica totalidad de los sectores económicos, nos da una idea de la

${ }^{6}$ Desde una perspectiva económica una de las definiciones del concepto de sostenibilidad más utilizadas en la literatura es la de Jack Pezzey (1992: 323) que define el término como la existencia de utilidad no decreciente de un miembro representativo de la sociedad durante milenios. PEZZEY, J., «Sustainability: An Interdisciplinary Guide». Environmental Values, 1(4), 1992, pp. 321-362.

7 KOETZ, T., et al., «Building better science-policy interfaces for international environmental governance: assessing potential within the Intergovernmental Platform for Biodiversity and Ecosystem Services». International environmental Agreements, DOI 10.1007/ s10784-011-9152-z, 2011.

8 Véase, por ejemplo, la teoría de la acción razonada de FISHBEIN, M., AJZEN, I., Belief, Attitude, Intention and Behaviour: An Introduction to Theory and Research, Reading, MA, Addison -Wesley, 1975. 
complejidad del reto al que nos enfrentamos. Este reto cuenta sin embargo con un aliado: los acuerdos ambientales internacionales.

Los acuerdos ambientales internacionales se definen como acuerdos legales entre estados. Su objetivo es la gestión de un recurso natural transnacional de manera conjunta y cooperativa. Así, se pasa de una situación no cooperativa de acciones individuales de los distintos países a una situación de cooperación y acción conjunta. Los acuerdos ambientales internacionales son de voluntario cumplimiento, por lo que es necesario que los países participantes puedan obtener beneficios tangibles de los mismos, si queremos que estos acuerdos sean efectivos en términos ambientales y duraderos 9 .

Las principales soluciones en las que se está trabajando a nivel internacional incluyen las negociaciones y acciones en el seno de la CMNUCC y en el seno del Protocolo de Kioto (PK). En el ámbito de la CMNUCC, y siguiendo la ruta de acción de Bali, se está trabajando en la cooperación a largo plazo con el fin de lograr: una reducción de emisiones que evite interferencias peligrosas con el clima, la adaptación a las nuevas condiciones climáticas, las transferencias financieras y las transferencias tecnológicas. Por otro lado, las negociaciones en el marco del PK se centran en los compromisos de reducción de emisiones de GEI de los países industrializados. De los principales hitos alcanzados, así como de los retos pendientes, se hablará más adelante.

Aunque el problema del cambio climático es por naturaleza multinivel (internacional, nacional, regional, local e individual) y concierne a todos los sectores, el presente artículo se centrará en el análisis a nivel internacional para analizar el papel de la Unión Europea en la gobernanza global del cambio climático. Las negociaciones dentro de la UE y a nivel internacional tienen elementos comunes en lo relativo a la complejidad de negociar entre países con distintos niveles de desarrollo, vulnerabilidad y capacidades. Así, tal y como argumentan Andrew Jordan, et al. ${ }^{10}$, la configuración de la UE hasta 2004 con más países «ricos» y contaminantes y la configuración actual, con países más y menos desarrollados (especialmente después de la ampliación en 2004), se puede ver como un laboratorio social o un microcosmos de los problemas y las soluciones a nivel global en materia climá-

${ }^{9}$ La existencia de beneficios netos no es el único factor que facilita los acuerdos ambientales internacionales. Véanse, entre otros, BARRETT, S., Why Cooperate? The Incentive to Supply Global Public Goods, Oxford University Press, Nueva York, 2007, BRETTEVILLEFROYN, C., «International Environmental Cooperation: The Role of Political Feasibility», en ATKINSON, G., et al., (eds.), Handbook of Sustainable Development, Edward Elgar, Cheltenham, 2007, o SANDLER, T., «Protocolo de Kioto: dificultades para la acción colectiva», Papeles de economía española, Economía y Cambio Climático, n. ${ }^{\circ}$ 121, 2009, pp. 14-24.

10 Vid. JORDAN, A., et al., op. cit., nota 4, p. 3. 
tica. Esto es así debido a que la UE-27 toma decisiones de manera conjunta, solucionando problemas de acción colectiva transfronterizos como el cambio climático. Por tanto, las negociaciones dentro de la UE pueden servir como ejemplo de cómo solucionar los problemas a escala global ${ }^{11}$ y pueden aportar información sobre los problemas que se presentan para la exitosa culminación de dichas negociaciones.

A continuación, se analizan las causas y las consecuencias del cambio climático para motivar el análisis posterior de la gobernanza de dicho fenómeno y el análisis del papel que juega la UE.

\section{Proceso, causas, causantes y consecuencias}

A nivel global sólo una docena de actores internacionales (incluyendo la UE) emiten la mayor parte de los GEI ${ }^{12}$. De hecho, más de la mitad de las emisiones globales son causadas por China, EEUU y la UE. Por tanto, cualquier acuerdo internacional en materia climática deberá contar, al menos, con los principales emisores de GEI para asegurar su efectividad en términos ambientales ${ }^{13}$. Es importante destacar sin embargo que con acuerdos parciales pueden darse diversos problemas. Entre los principales problemas destaca la posible falta de efectividad debido a que los países que se han unido al acuerdo no reducen las emisiones de GEI al nivel (global) deseado. Además, los países que no han ratificado el acuerdo pueden actuar como parásitos o free rider ${ }^{14}$, aumentando sus emisiones y reduciendo por tanto el efecto beneficioso del esfuerzo de los miembros que han ratificado el acuerdo.

La figura 1 detalla las emisiones actuales de gases de efecto invernadero de los principales países responsables expresados en términos porcentuales.

11 Para un análisis sucinto de la representación de la UE en las negociaciones climáticas internacionales véase ELOLA, T., «La representación de la UE en las negociaciones internacionales sobre el cambio climático tras la entrada en vigor del Tratado de Lisboa», Real Instituto Elcano, ARI 97/2011 - 25/05/2011, 2011.

12 MARÍN GONZÁLEZ, M., «Seguridad, Modelo Energético y Cambio Climático» en MINISTERIO DE DEFENSA (ed.), Seguridad, Modelo Energético y Cambio Climático, Cuadernos de Estrategia. Instituto Español de Estudios Estratégicos, n. ${ }^{\circ}$ 150, enero, 2011, pp. 15-25.

13 Otras variables para la evaluación de la política climática global son, sin embargo, necesarias desde una perspectiva racionalista. Así, HUITEMA, D., et al., detallan criterios adicionales como la eficiencia en términos de costes, la equidad, la legitimidad, la coordinación e integración con otras políticas y la concordancia con los principios legales existentes. HUITEMA, D., et al., «The evaluation of climate policy: theory and emerging practice in Europe», Policy Sciences, DOI 10.1007/s11077-011-9125-7, 2011.

${ }_{14}$ Véase ALCARÁZ VARÓ, E., HUGHES, B., Diccionario de términos económicos, financieros y comerciales, Barcelona, Arial Referencia, 2002. 


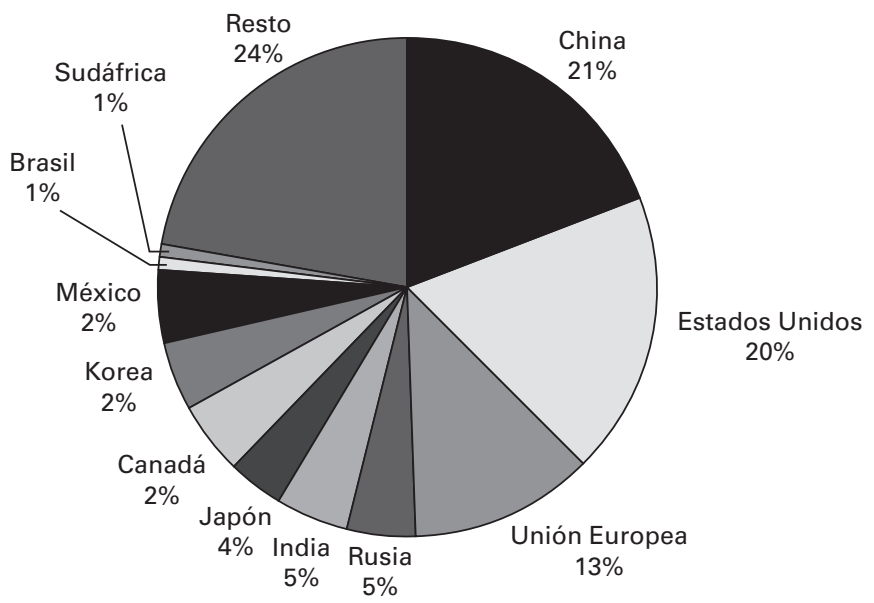

Fuente: Marín González (2011: 22).

\section{Figura 1}

Principales responsables de las emisiones de GEI a nivel global

Históricamente, los países desarrollados ${ }^{15}$, identificados en el PK y en la CMNUCC como países del anexo I, han emitido a la atmósfera la mayor parte de las emisiones de GEI. Nicholas Stern afirmaba que, desde 1850 hasta principios del siglo XXI, EEUU y Europa habían sido responsables

${ }^{15}$ La Convención Marco de acciones Unidas sobre el cambio Climático divide a los países en tres categorías. Por un lado están los países del anexo I que son países industrializados miembros de la OCDE en 1992 más las economías en transición y Rusia, los países bálticos, y varios países de Europa central y del este. Los países del anexo II por otro lado son los países del anexo I que pertenecen a la OCDE que deben procurar financiación a los países en desarrollo para que estos últimos mitiguen sus emisiones de gases de efecto invernadero y se adapten a las consecuencias del cambio climático. Además, los países del anexo II deben procurar que se llevan a cabo todas las acciones posibles para asegurar la transferencia tecnológica a los países con economías en transición y a los países en desarrollo. Esta visión es considerada sin embargo obsoleta en relación a ciertos países con economías en transición como China ya que en la actualidad la producción de aerogeneradores chinos y la producción de paneles solares chinos está convirtiéndose en una amenaza a la tradicional hegemonía europea y norteamericana en la materia. De hecho la Asociación Eólica Mundial aseguraba en 2010 que China ya era el líder mundial en lo relativo a capacidad eólica instalada (habiendo instalado 18,928 MW en un año, cifra que representa más de la mitad del mercado mundial en nuevas turbinas). Por último, los países no pertenecientes al anexo I son, en su mayoría, países en desarrollo especialmente vulnerables a las consecuencias del cambio climático, o países con un alto nivel de dependencia de los ingresos derivados de sus exportaciones de combustibles fósiles. Para ampliar la información consúltese: World Wind Energy Association (2010) y http:// unfccc.int/parties_and_observers/items/2704.php 
del $70 \%$ de las emisiones de gases de efecto invernadero ${ }^{16}$. El futuro de las emisiones de GEI sin embargo será determinado fundamentalmente por los países no pertenecientes al anexo I. Así, si bien en 1990 la UE y otros países del anexo I emitían más de la mitad de las emisiones de GEI a nivel mundial, las proyecciones realizadas por Naciones Unidas para mediados del presente siglo indican que los países en desarrollo emitirían aproximadamente tres cuartas partes de las emisiones de GEI. Un futuro acuerdo internacional, ambicioso, justo y vinculante, necesitará por tanto, la concurrencia de actores internacionales incluidos en el anexo 1 y no incluidos en dicho anexo.

La inclusión de los países menos desarrollados en los acuerdos climáticos deberá sortear los incentivos que tienen estos países para actuar como free riders. Estos incentivos se producen debido a tres efectos: el efecto del daño, el efecto del mercado energético y el efecto de la relación real de intercambio (terms of trade) ${ }^{17}$. Así, el efecto del daño implica que los países en desarrollo se benefician de la reducción de emisiones de los países que suscriben el acuerdo internacional, disminuyendo los incentivos de los free riders a contribuir a la provisión de un clima estable. El efecto del mercado energético implica que, al haber una reducción de GEI por parte de los países que suscriben el acuerdo internacional, se produce una bajada de los precios de los combustibles fósiles. Esta bajada de precios supone un incentivo para aumentar la demanda de dichos combustibles, aumentando así las emisiones de GEI. Por último, el efecto de la relación real de intercambio supone que al aumentar los precios de los bienes intensivos en GEI, las industrias que los producen se reubicarán en países en los que no haya obligación de reducir las emisiones de GEI.

El argumento de la pérdida de competitividad y de la posibilidad de deslocalizar la producción, es esgrimido por ciertas empresas que emiten GEI de manera intensiva para reducir sus obligaciones en materia de emisiones. Sin embargo, en general, los efectos de la deslocalización suelen ser limitados ${ }^{18}$ y dependerán de la industria analizada, de la magnitud de los costes ambientales, de la necesidad de estar cerca del cliente final, de la disponibilidad de mano de obra cualificada y de la prima de riesgo de realizar negocios en otros países, entre otros. Además, los países que suscri-

16 STERN, N., et al., The Economics of Climate Change. The Stern Review, Cambridge, Cambridge University Press, 2006.

17 BOSETTI, V., DECIAN, E., A gook opening. The key to make the most of multilateral action. Fondazione Eni Enrico Mattei, Documento de Trabajo presentado en EAERE 2011, Roma, 29 de junio - 2 de julio de 2011.

18 NEUMAYER, E., Greening Trade and Investment. Environmental Protection Without Protectionism, London, Earthscan, 2001. 
ben acuerdos climáticos más ambiciosos suelen proteger sus industrias de la competencia de los mercados internacionales y de la deslocalización empresarial, otorgándoles por ejemplo exenciones, o distribuyendo de manera gratuita derechos de emisión ${ }^{19}$. En la UE por ejemplo, tanto la industria del automóvil como las industrias intensivas en el uso de energía han ejercido una presión significativa y han conseguido limitar los efectos del paquete de energía y cambio climático sobre estos sectores.

En contraposición con el argumento anterior existen razones económicas para avanzar en la política climática. En 2008 el Programa de Naciones Unidas para el Medio Ambiente (PNUMA) ${ }^{20}$ cifraba el volumen del mercado global para productos y servicios ambientales en 1 billón de euros, y el número de personas empleadas en el sector de las energías renovables en 2,3 millones. Además se estima que el mercado global para productos y servicios ambientales se duplicará en la próxima década. La UE por su parte estima que el sector de la eco-innovación ${ }^{21}$ emplea a 3,4 millones de personas y representa, según la OCDE, aproximadamente el 2,5\% del PIB de la UE. La UE percibe por tanto las inversiones en innovación en el sector ambiental como parte de su estrategia para lograr una Europa competitiva y sostenible.

Centrándonos en la UE, las emisiones de GEI también se concentran mayoritariamente (más del 70\%) en seis Estados miembros que además son los más desarrollados (salvo Polonia). Así, el Reino Unido y Alemania aportan un tercio de los GEI de la UE-27, Italia y Francia aportan algo menos de un cuarto de las emisiones europeas y España y Polonia un $8 \%$ cada una ${ }^{22}$.

En relación a los sectores causantes de las emisiones de GEI a nivel global, las emisiones «energéticas», incluyendo la producción de energía, los usos industriales, el transporte o los edificios ${ }^{23}$, suponían a principios del presente siglo el 65\% de dichas emisiones. La mitigación del cambio climático por tanto llevará implícita una transformación energética sin precedentes desde la revolución industrial. El 35\% restante de los gases de efecto in-

19 COM (2010) 265. Comunicación de la Comisión al Parlamento Europeo, al Consejo, al Comité Económico y Social europeo y al Comité de las regiones, Análisis de las opciones para rebasar el objetivo del $20 \%$ de reducción de las emisiones de gases de efecto invernadero y evaluación del riesgo de fugas de carbono, 26 de mayo de 2010.

20 Basándose en el estudio de Berger, véase UNEP, Green Jobs Report. Some Facts and Figures and Highlights, 2008.

${ }^{21}$ En general se define el término eco-innovación como las transformaciones en productos, procesos, y servicios resultan en menores impactos sobre el entorno.

22 European Environment Agency, (EEA), Annual EC greenhouse gas inventory 19902006 and Inventory Report, EEA Technical Report 6/2008, Copenhagen, European Environment Agency, 2008.

${ }^{23}$ El US Department of Energy, proporciona datos desglosados de las emisiones de GEI de los edificios. Véase US DoE, 2010 Buildings Energy Data Book. Energy Efficiency and Renewable Energy. March 2011, 2011. 
vernadero provenía de la gestión de residuos, los usos agrícolas o el cambio en el uso de la tierra (principalmente la deforestación) ${ }^{24}$. En 2004 la aportación específica de cada uno de estos sectores se muestra en la figura 2.

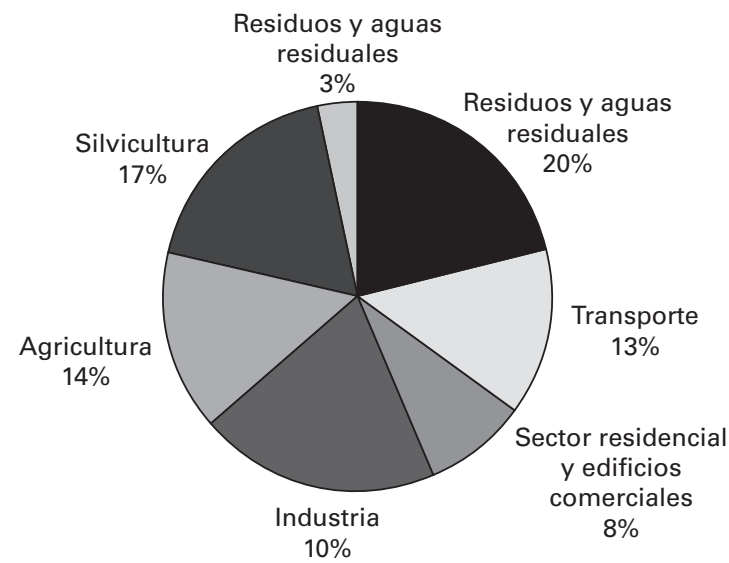

Fuente: IPCC (2007: figura 2.1) 25

Figura 2

Emisiones Mundiales de Gases de Efecto Invernadero por sectores (2004)

En la UE-2726 la composición de las emisiones de GEI en 2007 (expresado en porcentaje) según los sectores es la siguiente: las industrias energéticas $(31,9 \%)$, las industrias no energéticas como las manufacturas, la construcción y los procesos industriales $(21,3 \%)^{27}$, el transporte excluyendo los «búnker ${ }^{28}$ » internacionales $(19,5 \%$, siendo el único sector que ha experimentado un aumento ininterrumpido en sus emisiones desde 1990), el sector residencial $(8.5 \%)$, los sectores comercial e institucional (3,3\%), la agricultura, la silvicultura y la pesca $(10,6 \%)$ y otros $(5 \%)$.

24 Vid. STERN, N., et al., op. cit., nota 16, p. 8.

25 Disponible en inglés en: http://www.ipcc.ch/publications_and_data/ar4/syr/en/ figure-2-1.htmlyenespañolen:http:/www.marm.es/es/ceneam/recursos/mini-portales-tematicos/ 090471228009689d_tcm7-12934.pdf

$26 \mathrm{http} / /$ ec.europa.eu/energy/publications/doc/statistics/ext_greenhouse_gas_emissions_ by_sector.pdf

${ }^{27}$ Incluyen las emisiones provenientes de la quema de combustibles fósiles para usos agrícolas, forestales y pesqueros, otras emisiones «fugitivas» no incluidas en el resto de apartados provenientes de disolventes, uso de productos agrícolas y residuos.

${ }^{28}$ Es decir, el tráfico internacional aéreo y marítimo. 
En relación a las consecuencias de las emisiones de gases de efecto invernadero los datos disponibles a nivel global indican que, desde principios del siglo XX hasta la primera mitad de la primera década del siglo XXI, la temperatura de la tierra ha aumentado en $0,74^{\circ} \mathrm{C}$ y el aumento en el nivel del mar durante el siglo pasado ha sido de $17 \mathrm{~cm}$. En Europa el aumento de temperaturas ha sido mayor, llegando a $1^{\circ} \mathrm{C}$. El IPCC ${ }^{29}$ detalla en el último informe disponible (Cuarto Informe de Evaluación de 2007) que las consecuencias a nivel mundial serán más graves cuanto mayores sean los aumentos en las temperaturas y que afectarán en mayor medida a los países menos desarrollados. Dichos países, además de no haber sido los principales responsables en el pasado del cambio climático de origen antropogénico, disponen de menos medios para adaptarse a él.

El informe de Nicholas Stern ${ }^{30}$ también es claro en cuanto a las consecuencias del cambio climático. En este informe se expone que para latitudes más altas, un aumento en las temperaturas podría ser beneficioso al aumentar la productividad agrícola, disminuir las necesidades de calefacción o aumentar el atractivo turístico en países como Rusia, Canadá o los países escandinavos. Sin embargo, para países situados más al sur se pueden esperar reducciones en la productividad agrícola y en la disponibilidad de agua del $20 \%$.

En Europa, las consecuencias esperadas del cambio climático según el IPCC $^{31}$ incluyen una mayor frecuencia y gravedad de fenómenos meteorológicos extremos, como por ejemplo: sequías, inundaciones costeras e interiores (derivadas del desbordamiento de ríos) así como un aumento de la erosión del suelo. Se esperan a su vez menos precipitaciones en forma de nieve, una reducción de los glaciares y pérdidas muy significativas de biodiversidad, especialmente en escenarios de mayores aumentos de temperaturas (y emisiones). La situación en países del sur de Europa, como España, es sin duda más grave que en los países del norte de Europa ya que en el sur la escasez de agua y la vulnerabilidad al cambio climático es más acusada. Esta menor disponibilidad de agua a su vez supondrá una menor oferta de energía hidráulica que afectará a las emisiones de GEI, si no se cubre esta disminución de energía hidráulica con aumentos en la producción de energía con fuentes energéticas de bajas emisiones de $\mathrm{CO}_{2}$.

El confort térmico en verano puede además disminuir en los países del sur de Europa, por lo que los periodos en los que los turistas visitan España

29 IPCC es el acrónimo de Intergovernmental Panel on Climate Change o panel intergubernamental de expertos sobre cambio climático, que recopila y sintetiza el conocimiento existente evaluado por pares relativo al cambio climático.

30 Vid. STERN, N., et al., (2006), op. cit., nota 16, p. 8.

31 IPCC, Climate Change 2007, Synthesis Report, 2007. 
y los lugares de visita pueden verse alterados. La aportación del sector turístico al PIB español está en el entorno del $10 \%$ a lo largo de la primera década del siglo XXI. Por ello las acciones en materia de mitigación y adaptación al cambio climático serán sin duda una prioridad estratégica a medio y largo plazo. Otros sectores, como el de la agricultura también se verán significativamente afectados si las predicciones se cumplen. Así, el IPCC estima que en el sur de Europa habrá disminuciones en la productividad de los cultivos agrícolas. Finalmente, la mayor frecuencia y gravedad de olas de calor, como la que asoló Europa en 2003, especialmente a Francia, pueden repetirse con mayor frecuencia, afectando a la población más vulnerable.

El IPCC estima que la estabilización de las concentraciones de GEI entre $445 \mathrm{ppm}$ y $710 \mathrm{ppm}$ puede suponer variaciones en el PIB que oscilarían entre un aumento del PIB del 1\% y una disminución del 5,5\%.

Para el escenario de estabilización más estricto, (445-535 ppm de $\left.\mathrm{CO}_{2} \mathrm{e}\right)$ nos enfrentaríamos, a nivel global, a un promedio de ralentización de la tasa de crecimiento del PIB de menos de un $0,12 \%$ del anual. Véase la tabla 1 para un mayor detalle de las estimaciones de costes.

\section{Tabla 1}

Costes macroeconómicos mundiales estimados en 2030 y en 2050

\begin{tabular}{|c|c|c|c|c|c|c|}
\hline \multirow[t]{2}{*}{$\begin{array}{l}\text { Niveles de } \\
\text { estabilización } \\
\text { (ppm de } \\
\mathrm{CO}_{2} \text {-eq) }\end{array}$} & \multicolumn{2}{|c|}{$\begin{array}{c}\text { Mediana de } \\
\text { reducción } \\
\text { del PIB a) }(\%)\end{array}$} & \multicolumn{2}{|c|}{$\begin{array}{c}\text { Intervalo de valores } \\
\text { de reducción del PIB b) }(\%)\end{array}$} & \multicolumn{2}{|c|}{$\begin{array}{l}\text { Reducción de las } \\
\text { tasas de crecimiento } \\
\text { promedias anuales } \\
\text { del PIB (en puntos } \\
\text { porcentuales) }\end{array}$} \\
\hline & 2030 & 2050 & 2030 & 2050 & 2030 & 2050 \\
\hline $445-535^{\mathrm{d})}$ & \multicolumn{2}{|c|}{ No disponible } & $<3$ & $<5,5$ & $<0,12$ & $<0,12$ \\
\hline $535-590$ & 0,6 & 1,3 & $\begin{array}{l}\text { entre } 0,2 \text { y } \\
2,5\end{array}$ & $\begin{array}{l}\text { entre } \\
\text { ligeramente } \\
\text { negativo } \\
\text { y } 4\end{array}$ & $<0,1$ & $<0,1$ \\
\hline $590-710$ & 0,2 & 0,5 & $\begin{array}{l}\text { entre }-0,6 \\
\text { y } 1,2\end{array}$ & entre -1 y 2 & $<0,06$ & $<0,05$ \\
\hline
\end{tabular}

Fuente: IPCC (2007: 22) 32

${ }^{32}$ Los valores indicados en esta tabla corresponden al conjunto de publicaciones científicas respecto de todos los valores de referencia y escenarios de mitigación que arrojan valores numéricos del PIB. 
Tanto las causas y los causantes del cambio climático, como las consecuencias esperadas y los costes de reducir las emisiones de GEI, hacen que el cambio climático haya sido considerado a nivel internacional como el mayor fallo de mercado y la gran amenaza del siglo XXI junto con la pobreza $^{33}$.

La responsabilidad histórica de la UE en materia de emisiones, las consecuencias esperadas y el deseo de la UE de ser un líder global en materia climática proporcionan un escenario propicio para que la UE desempeñe un papel fundamental en la gobernanza climática global.

\section{La gobernanza global del cambio climático y la UE}

A nivel internacional, el problema del cambio climático es complejo de solucionar por tres razones principales ${ }^{34}$. La primera razón es, que aun considerando el cambio climático como un experimento de proporciones planetarias, y, aun siendo posible la interferencia peligrosa con el clima, la supervivencia humana no está en juego de manera inminente. El incentivo para la acción es por tanto menor que si estuviéramos ante un fenómeno que amenazara la supervivencia a corto plazo. Además, sabemos que distintos países se ven afectados por el cambio climático de distinta manera lo cual implica tener incentivos asimétricos para la acción. En tercer lugar, la reducción de emisiones de GEI va a requerir inversiones y cambios en nuestros sistemas de producción, distribución y consumo. Estas inversiones implican asumir el coste de oportunidad de no invertir en otros proyectos que pueden ser igualmente deseables a nivel social. Todo ello dificulta la acción en materia de reducciones de GEI.

El deseo de liderazgo europeo en materia climática comenzó a fraguarse a finales de la década de los 80 del siglo Xx. Dicho deseo ha estado

a) PIB mundial basado en los tipos de cambio del mercado.

b) Se indican, cuando procede, el percentil 10 y 90 de los datos analizados. Los valores negativos indican un aumento del PIB. En la primera fila (445-535 ppm de $\mathrm{CO}_{2}$-eq) se indica la estimación de la cota superior según los artículos publicados únicamente.

c) La reducción de la tasa de crecimiento anual se calcula en base al promedio de la reducción que, durante el período estudiado, daría lugar a la disminución del PIB señalada de aquí a 2030 y a 2050 , respectivamente.

d) El número de estudios es relativamente pequeño y sus valores de referencia suelen ser bajos. Unos valores de referencia altos suelen entrañar un coste más elevado.

e) Estos valores corresponden a la estimación más alta de la reducción del PIB, columna 3.

33 STERN, N., «Managing Climate Change and Overcoming Poverty: Facing the Realities and Building a Global Agreement», London, London School of Economics, 2009.

${ }^{34}$ Vid. BARRETT, S., op. cit., nota 9, p. 5. 
apoyado por una política exterior europea que pretendía construir y afianzar sus alianzas con otros países. Adicionalmente, la UE ha querido diferenciarse de EEUU subrayando su faceta de poder civil que lidera mediante el ejemplo. Además, el apoyo de los ciudadanos a una mayor protección ambiental ${ }^{35}$ es parte integral de los valores europeos, especialmente de la UE15. Este apoyo ciudadano ha facilitado la acción en materia ambiental así como en materia climática de la UE. La política climática de la UE ha tenido también como objetivo la protección de la población de las consecuencias del cambio climático. Asimismo, la UE ha querido beneficiarse de la oportunidad económica que ofrece, por ejemplo, la eco-innovación y, en especial, la innovación en materia energética. Un motivo adicional para el desarrollo de la política climática es el aumento de la seguridad energética, al permitir una mayor penetración de energías renovables en el mix energético, reduciendo la dependencia de los combustibles fósiles importados. Por último, el potencial de creación de empleos ha impulsado también la política ambiental europea. Dicho potencial ha sido cifrado en 6 millones de puestos de trabajo potenciales en la UE si se eleva el compromiso de reducción de emisiones del $20 \%$ al $30 \%$ en $2020^{36}$.

Sin embargo, las demandas de los principales actores internacionales como China o EEUU, el propio desarrollo de las negociaciones internacionales y el peso relativo decreciente de la UE como uno de los principales emisores de GEI, han dificultado el camino hacia el liderazgo climático europeo. A todo ello hay que añadir las posiciones negociadoras divergentes en el seno de la UE.

Dentro de la UE, existen también diversos problemas para reducir las emisiones de GEI. En primer lugar, la UE quiere liderar la lucha contra el cambio climático siendo un sistema de gobernanza sin un único líder que gobierne. En segundo lugar, la falta de implementación de algunas de las medidas de política climática. Un ejemplo reciente de esto último es la distancia entre los objetivos de eficiencia energética y lo conseguido, tal y como refleja la reciente propuesta de la Directiva sobre Eficiencia Energética $^{37}$. Por último, la UE quiere afrontar las negociaciones climáticas in-

35 Véase por ejemplo el euro barómetro 75.2 de mayo de 2011 o el análisis de GERHARDS, J., LENGFELD, H., «The growing remit of the EU in environmental and climate change policy and citizens' support across the Union», European Journal of Social Policy, 2008 .

${ }^{36}$ Bajo los supuestos del modelo usado por JAEGER, C., et al., A New Growth Path for Europe. Generating Prosperity and Jobs in the Low-Carbon Economy. Synthesis Report, European Climate Forum, 2011.

${ }^{37} \operatorname{COM}(2011) 370$ final. Proposal for a Directive of the European Parliament and of the Council on energy efficiency and repealing Directives 2004/8/EC and 2006/32/EC, 22 de junio de 2011. 
ternacionales de manera unida, pero se enfrenta a posturas divergentes entre sus miembros. Aunque las razones para reducir las emisiones de GEI no siempre han tenido como transfondo el cambio climático ${ }^{38}$, tradicionalmente ha habido dentro de Europa líderes climáticos como los Países Bajos, Dinamarca o Alemania que desde finales de los 80 establecieron reducciones de las emisiones de GEI más estrictas que otros países de la UE. A este grupo inicial de líderes se unieron más adelante el Reino Unido o Austria aceptando por ejemplo las reducciones de GEI acordadas bajo el enfoque Triptych $^{39}$.

Frente a los líderes europeos hay un grupo más rezagado que tradicionalmente ha incluido a países menos desarrollados y países con mayor dependencia de los combustibles fósiles en su matriz energética. Así, España, Portugal, Irlanda o Grecia fueron, en las primeras etapas de las negociaciones climáticas, los rezagados, apoyando reducciones de emisiones a nivel de la UE siempre que estas reducciones se distribuyeran internamente teniendo en cuenta sus condiciones de desarrollo. A modo de ilustración, la tabla 2 muestra los objetivos de la UE-15 para el primer periodo de compromiso del PK de la UE.

Los países que pasaron a formar parte de la UE en 2004, como Polonia, han pasado a ser los países rezagados en materia climática. Un buen ejemplo de ello es la postura polaca en las negociaciones climáticas recientes que han tenido lugar en la UE. Polonia ha bloqueado la propuesta de aumentar el grado de compromiso de reducción de emisiones de GEI de la UE en junio de $2011^{40}$. Junto con este bloqueo se produjeron además declaraciones por parte del comisario polaco designado para el área de Presupuestos, Janusz Lewandowski, que cuestionaban el cambio climático y señalaban que para Polonia sería muy costoso prescindir del carbón como fuente energética.

38 Véase por ejemplo el papel de la organización de la cumbre climática en Bonn en 1995, la ascensión de la coalición «roji-verde» o la reconversión industrial en la postura de negociación climática de Alemania, tradicionalmente más avanzada. En general, factores como la estructura económica de los países de la UE, la composición de la matriz energética, los shocks externos (crisis económicas y la evolución de los mercados del petróleo), la innovación tecnológica, las posiciones de otros grandes contaminadores y el liderazgo intelectual (de Tony Blair, Angela Merkel, Nicolás Sarkozy, Jaques Delors o José Manuel Durao Barroso, por ejemplo), han influido de manera significativa en la agenda climática de la UE.

39 Tryptich se refiere al método elaborado en la universidad de Utrech para la asignación de las cantidades de GEI dentro de la UE durante el primer periodo de compromiso del PK. Vid. JORDAN, A., et al., op. cit., nota 4, p. 3.

$40 \mathrm{http} / / / \mathrm{www}$.euractiv.com/en/climate-environment/polands-eu-commissioner-surpriseclimate-denial-move-news-505869?utm_source=EurActiv+Newsletter\&utm_campaign =d8c70d6adc-my_google_analytics_key\&utm_medium=email 


\section{Tabla 2}

Variación de emisiones de GEI UE-15

(1990-2012) en términos porcentuales

\begin{tabular}{l|r}
\hline \multicolumn{1}{c|}{ País } & Asignación acordada \\
\hline Austria & -13 \\
Bélgica & $-7,5$ \\
Dinamarca & -21 \\
Finlandia & 0 \\
Francia & 0 \\
Alemania & -21 \\
Grecia & +25 \\
Irlanda & +13 \\
Italia & $-6,5$ \\
Luxemburgo & -28 \\
Países Bajos & -6 \\
Portugal & +27 \\
España & +15 \\
Suecia & +4 \\
Reino Unido & -12.5 \\
UE-15 & -8 \\
\hline
\end{tabular}

Fuente: Adaptado de la decisión del Consejo del 25 de abril de 2002 (2002/358/CE).

Con todo lo expuesto observamos que, a medida que los esfuerzos de reducción y los costes de reducción de GEI aumentan, es previsible que las divergencias en el seno de la UE, al igual que a nivel internacional, se hagan más visibles y dificulten el liderazgo europeo.

La definición de liderazgo, sin embargo, no siempre se usa con preci$\operatorname{sión}^{41}$. Una de las definiciones disponibles en la literatura es la de Unde$\mathrm{ral}^{42}$ que define el concepto de liderazgo como «una relación asimétrica de influencia en la que un actor guía o dirige el comportamiento de otros en pro de la consecución de un objetivo determinado durante un cierto periodo de tiempo» ${ }^{43}$. Para entender el papel de la UE en la gobernanza global del cambio climático y evaluar de manera sistemática el «liderazgo» de la UE

${ }^{41}$ ANDERSEN, S., AGRAWALA, S., «Leaders, Pushers and Laggards in the Making of the Climate Regime». Global Environmental Change, 12, 2002, pp. 41-51.

42 UNDERAL, A., «Solving Collective Problems: Notes on Three Modes of Leadership» en Challenges of a Changing World: Festchrift to Willy Østreng, 139-153. Lysaker, Norway: The Fridtjof Nansen Institute, 1991.

43 Traducción de la autora. 
este apartado analizará los distintos tipos de liderazgo en materia climática ${ }^{44},{ }^{45},{ }^{46},{ }^{47}$. En resumen, estos tipos de liderazgo se pueden clasificar en la tabla 3 .

Además de lo anterior es necesario tener en cuenta que el liderazgo internacional (conformado por el potencial de guía de un actor sumado a la motivación para guiar a otros actores), añadido a las habilidades negociadoras y de convicción, conforman los elementos esenciales para la influencia en los procesos de negociación del clima ${ }^{48}$. Si alguno de los elementos anteriormente mencionados no está presente, podemos tener un liderazgo sin influencia real en los resultados de las negociaciones.

Una vez presentados los conceptos teóricos relacionados con el liderazgo, es posible argumentar que la UE ha ejercido un liderazgo direccional basado en tres elementos principales: la presión negociadora, los instrumentos de política climática utilizados y las acciones emprendidas en el seno de la UE49.

En primer lugar la presión y la negociación en momentos concretos del proceso negociador. En lo referente a los momentos clave en relación al liderazgo de la UE, parece haber un consenso razonable entre los expertos en que estos hitos son varios. En primer lugar, el papel jugado por la UE en la CMNUCC en la que la UE ayudó a fortalecer la convención. En segundo lugar, el hecho de que la UE ayudó de manera decisiva a «salvar» el proceso negociador en 2001 tras la no ratificación de EEUU del PK. En tercer lugar, la entrada en funcionamiento del PK gracias a las negociaciones con Rusia (en las que la UE apoyó la entrada de Rusia en la $\mathrm{OMC}^{50}$ a cambio de la ratificación rusa del Protocolo de Kioto). Dicha ratificación hizo que se cumplieran las dos condiciones para la entrada en vigor del Protocolo de Kioto ${ }^{51}$. En cuarto lugar, la CdP15 en la que, a pesar del compromiso unilateral de reducción de emisiones del $20 \%$ en relación a 1990, la UE se vio relegada a un segundo plano en las etapas finales de la cumbre.

44 Vid, ANDERSEN, S., AGRAWALA, S., op. cit., nota 41, p. 17.

45 SKOVDIN, T., ANDERSEN, S., «Leadership Revisited». Global Environmental Politics, Volume 6, Number 3, 2006, pp. 13-27.

${ }^{46}$ ORBERTHÜR, S., ROCHE KELLY, C., «EU Leadership in International Climate Policy. Achievements and Challenges». The International Spectator. Vol. 43, 2008, pp. 35-50.

${ }^{47}$ OBERTÜR, S., «Global Climate Governance after Cancun: options for EU leadership». The International Spectator. Vol. 46, 2011, pp. 5-13.

48 Vid. SKOVDIN, T., ANDERSEN, S., op. cit., nota 45, p. 17.

49 Vid. JORDAN, A., et al., op. cit., nota 4, p. 3.

50 OMC es el acrónimo de Organización Mundial del Comercio.

${ }^{51}$ Que 55 países o más que supusiesen el 55\% de las emisiones globales hubieran ratificado el Protocolo de Kioto. 


\section{Tabla 3}

Tipos de liderazgo, agentes, fases negociadoras y características

\begin{tabular}{|c|c|c|c|}
\hline $\begin{array}{c}\text { Tipos de } \\
\text { liderazgo }\end{array}$ & $\begin{array}{l}\text { Agentes que } \\
\text { ejercen el } \\
\text { liderazgo }\end{array}$ & $\begin{array}{l}\text { Fases negociadoras } \\
\text { (Años en las que se } \\
\text { observa de manera } \\
\text { más marcada el tipo de } \\
\text { liderazgo en el ámbito } \\
\text { climático) }\end{array}$ & Características \\
\hline Intelectual & $\begin{array}{l}\text { Generalmente } \\
\text { individuos }\end{array}$ & $\begin{array}{l}\text { Estableciendo la } \\
\text { agenda negociadora } \\
\text { Agenda-setting } \\
(1950-1991)\end{array}$ & $\begin{array}{l}\text { El líder intelectual } \\
\text { produce conocimiento } \\
\text { que afecta a las partes } \\
\text { involucradas en el proceso } \\
\text { negociador. }\end{array}$ \\
\hline Instrumental & $\begin{array}{l}\text { Individuos o } \\
\text { instituciones }\end{array}$ & $\begin{array}{l}\text { Estableciendo la } \\
\text { agenda negociadora } \\
\text { Agenda-setting } \\
(1950-1991)\end{array}$ & $\begin{array}{l}\text { El líder instrumental } \\
\text { usa las ideas del } \\
\text { líder intelectual } \\
\text { amplificándolas para } \\
\text { influir en la agenda de } \\
\text { negociación. }\end{array}$ \\
\hline $\begin{array}{l}\text { Estructural o } \\
\text { basado en el } \\
\text { poder }\end{array}$ & Estados & $\begin{array}{l}\text { Tanto en las decisiones } \\
\text { sobre la agenda de } \\
\text { negociación } \\
(1950-1991) \text { como en } \\
\text { las negociaciones } \\
(1991-1997)\end{array}$ & $\begin{array}{l}\text { Se basa en la habilidad } \\
\text { del estado de ejecutar } \\
\text { amenazas y de cumplir } \\
\text { con sus compromisos. } \\
\text { Requiere que además } \\
\text { del interés del estado se } \\
\text { busque el interés común. }\end{array}$ \\
\hline Direccional & Estados & $\begin{array}{l}\text { Negociación } \\
(1991-1997) \text { y } \\
\text { puesta en práctica } \\
\text { (1997-actualidad) }\end{array}$ & $\begin{array}{l}\text { El líder dará ejemplo } \\
\text { a través de las propias } \\
\text { acciones y sacrificios } \\
\text { de cómo solucionar los } \\
\text { problemas. Puede darse } \\
\text { bien porque el líder es } \\
\text { el primero en actuar } \\
\text { o bien porque el líder } \\
\text { demuestra a los otros que } \\
\text { una solución es superior } \\
\text { a otras. }\end{array}$ \\
\hline
\end{tabular}

Fuentes: Adaptado de Skodvin y Andersen (2006), Andersen y Agrawala (2006).

En segundo lugar, la puesta en práctica de instrumentos de política ambiental novedosos. El ejemplo más claro aquí es la puesta en marcha desde el 2005 del mercado europeo de emisiones, idea formulada originalmente 
por EEUU y rechazada en un principio por las $\mathrm{ONG}^{52}$ y por Europa. Con el mercado europeo de emisiones la EU ha demostrando que se puede crear un mercado transnacional de $\mathrm{CO}_{2}$. Este mercado de emisiones ha mostrado un posible camino a seguir a nivel internacional en materia de intercambio de derechos de emisión de GEI. Esto es así a pesar de las asignaciones iniciales excesivas de derechos de emisión, de las limitaciones en cuanto a los sectores incluidos, de los fallos de seguridad y de la volatilidad derivada de la incertidumbre relativa a la evolución de la economía, entre otros.

En tercer lugar, la acción dentro de las fronteras de la UE, a través del diseño de programas y la adquisición de compromisos de manera unilateral. Aquí los programas europeos de cambio climático, así como el compromiso de reducir las emisiones en un $20 \%$ en 2020, son ejemplos de la acción emprendida por la UE y que refleja el liderazgo direccional. Además, según Andrew Jordan ${ }^{53}$, la UE ha logrado un papel preponderante en las negociaciones internacionales en lo relativo a la adaptación y las fuentes de financiación para los países en desarrollo. Por último, la UE ha ayudado a difundir principios ambientales como el principio de cautela ${ }^{54}$.

\section{La política climática de la UE}

La política climática de la UE se ha caracterizado por intentar aportar un enfoque integral en el que los compromisos ambientales se justificaran en términos de costes, sin olvidar la equidad, el apoyo ciudadano y la oportunidad política. Este subapartado analiza algunos de los elementos clave en la política climática europea en materia de mitigación, adaptación y transferencias de fondos y tecnología. El subapartado finaliza haciendo un breve repaso de las principales etapas en el desarrollo de la política ambiental. Este repaso muestra una evolución desde la práctica inexistencia de políticas climáticas al desarrollo de una suma de políticas individuales, para llegar en los últimos años del siglo XX y principios del XXI a una política climática europea.

En relación al apartado de mitigación se ha puesto el énfasis en la seguridad energética, el aumento de la competitividad y la eficiencia ener-

52 ONG es el acrónimo de Organizaciones No Gubernamentales.

53 Vid. JORDAN, A., et al., op. cit., nota 4, p. 3.

${ }^{54}$ El principio de cautela (precautionary principle) se reconoció a nivel internacional en la Asamblea General de Naciones Unidas en 1982 y se incorporó a la declaración de Río de Janeiro en 1992. El principio de precaución establece que, si existe la posibilidad de sufrir daños serios o irreversibles, la ausencia de certidumbre científica absoluta no será una razón aceptable para la inacción. 
gética ${ }^{55}$. El hecho de que las medidas de eficiencia energética sean las más rápidas de implementar y eficientes en términos de costes, en principio, las hace atractivas en un contexto de crisis económica. Sin embargo, quizá porque son las medidas menos «visibles» y por el contexto económico adverso, los compromisos europeos en este ámbito no se están cumpliendo. De hecho, la propuesta de 22 de junio de 2011 para una nueva Directiva sobre Eficiencia Energética afirma que las últimas estimaciones de la Comisión apuntan a que las medidas actuales de los Estados miembros sólo alcanzarán la mitad del objetivo propuesto en materia de ahorro y eficiencia energética ${ }^{56}$. Con el fin de avanzar en las medidas más eficientes para reducir las emisiones de GEI, el gráfico 1 a continuación aporta información sobre el coste marginal de distintas medidas de mitigación. Las medidas de eficiencia energética en la envolvente de los edificios, en el uso de la calefacción, en el consumo de agua así como la producción de motores más eficientes en los vehículos, son las que aportarían beneficios netos.

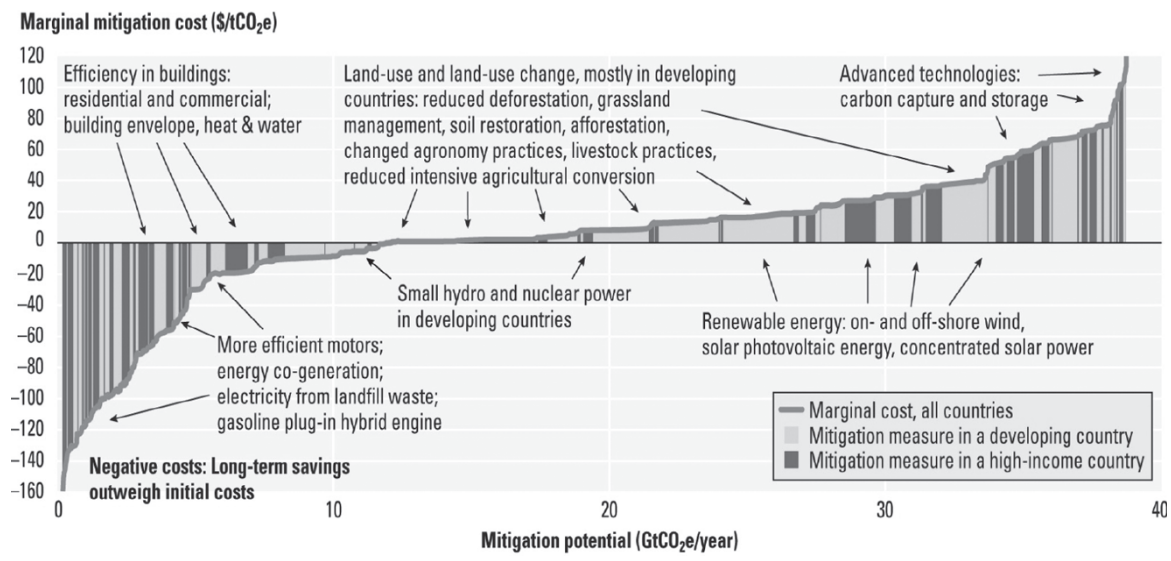

Fuente: McKinsey \& Company 2009 y World Development Report 2010.

\section{Gráfico 1}

Sectores o actividades (eje de abscisas) y costes marginales de mitigación (eje de ordenadas)

55 Véase HOBLEY, A., ADAMS, D., «The EU and Climate Change in the lead up to Cancun: Impacts, policies and Positions». Real Instituto Elcano. ARI 166/210. 24/11/2010, 2010.

$56 \operatorname{COM}(2011) 370$ final, Proposal for a Directive of the European Parliament and of the Council on energy efficiency and repealing Directives 2004/8/EC and 2006/32/EC, 22 de junio de 2011. 
En materia de adaptación, el desarrollo de las políticas climáticas ha sido tardío, especialmente en los países desarrollados, en los que se ha asumido históricamente una adaptación espontánea suficiente, fruto de un mayor desarrollo económico. La UE ha adoptado, en lo que respecta a la adaptación, un enfoque gradual a través de su Libro Blanco sobre adaptación ${ }^{57}$. Así, hasta 2012 se investigarán y compartirán conocimientos relativos a las consecuencias del cambio climático para la UE, se hará un esfuerzo de integración de las políticas de adaptación en otras áreas de la política europea, se usarán de manera complementaria los distintos instrumentos de regulación y de mercado y, finalmente, se redoblarán los esfuerzos en los relativo a la cooperación internacional.

En lo relativo al capítulo de transferencias de capital para la mitigación y la adaptación de los países en desarrollo, la UE, tras el Acuerdo de Copenhague, se ha comprometido con más de un tercio de toda la ayuda comprometida entre 2010 y 2012 de los países desarrollados. Si comparamos la responsabilidad histórica de la UE-25 en la emisión de GEI hasta 2007 (calculada en un 26-28\%, dependiendo de los supuestos ${ }^{58}$ ) con la aportación de fondos para la mitigación y la adaptación de países desarrollados, también se puede argumentar que, en lo relativo a las transferencias de fondos, la UE ejerce un liderazgo direccional basado en el ejemplo y en el esfuerzo propio.

Es sabido que uno de los pilares básicos en la lucha contra el cambio climático es el desarrollo de nuevas tecnologías y la innovación en productos y procesos. En lo referente a estas variables la UE ha desarrollado desde mediados de la pasada década un plan de acción para las tecnologías ambientales que se ha apoyado en la comercialización de nuevas tecnologías a nivel global y en la mejora de las condiciones de mercado ${ }^{59}$. De hecho, en el periodo 2008-2013 la UE va a destinar aproximadamente 200 millones de euros a financiar proyectos que estimulen el desarrollo de la eco-innovación ${ }^{60}$.

Desde una perspectiva temporal, si analizamos las distintas etapas de la política climática de la UE, se podría decir que el desarrollo de dicha política se ha producido en seis etapas ${ }^{61}$. Con anterioridad a 1988 hay actividades de I+D y hay una conciencia científica emergente en relación al problema del cambio climático. No hay sin embargo un desarrollo significativo

$57 \operatorname{COM}(2009) 147$ final, White Paper. Adapting to climate change: towards a European framework for action, 1 de abril de 2009.

58 DELLINK, D., et al., «Sharing the burden of financing adaptation to climate change». Global Environmental Change. 19, 2009, pp. 411-421.

59 Vid. HOBLEY, D., ADAMS, D., op. cit., nota 55, p. 20.

${ }^{60} \mathrm{http} / / / \mathrm{ec}$. europa.eu/environment/eco-innovation/index_en.htm

61 Vid. JORDAN, A., et al., op. cit., nota 4, p. 3. 
y global de políticas climáticas durante este periodo ya que no hay apoyo por parte de los Estados miembros. De 1988 a 1992 surge el primer intento de liderazgo europeo en materia climática, se firma la CMNUCC y fracasa el intento de establecer un impuesto sobre la energía y el $\mathrm{CO}_{2}$. Desde 1992 a 1997, aun siendo un periodo marcado por la Cumbre de la Tierra de Río y la firma del Protocolo de Kioto, dentro de Europa se da un periodo de relativa falta de coordinación en relación al cambio climático, así como una brecha entre los objetivos a los que se compromete la UE (limitar el aumento de las temperaturas a $2^{\circ} \mathrm{C}$ en relación a las temperaturas de la etapa preindustrial) y las acciones acometidas para lograr dicho objetivo.

De 1997 a 2001 se da el segundo intento en materia de liderazgo europeo en relación al cambio climático. Durante este periodo se establecen los acuerdos de reparto de carga para el primer periodo de compromiso del PK en el que la UE acepta los compromisos más ambiciosos dentro del grupo de los países del anexo B. Además se desarrolla el primer programa europeo sobre cambio climático (First European Climate Change Program o ECCP) encaminado a desarrollar medidas efectivas para el cumplimiento de los objetivos de Kioto en términos ambientales y eficientes en términos de costes. Entre 2001 y 2005 se da una expansión y fortalecimiento del marco regulatorio de la UE en lo relativo al cambio climático con la ratificación del Protocolo de Kioto, el desarrollo normativo del mercado europeo de emisiones, las negociaciones con Rusia y, finalmente, la entrada en vigor del Protocolo de Kioto el 16 de febrero de 2005.

La última etapa se extiende desde el 2005 a la actualidad. Los elementos más reseñables incluyen los esfuerzos realizados para cumplir con Kioto y las dificultades para acordar la arquitectura post 2012. En primer lugar, nos encontramos con la adopción por parte del Consejo de un objetivo de reducción de emisiones del $20 \%$ en 2020 en relación a las emisiones de 1990. Además, se acuerda aumentar el uso de las energías renovables hasta alcanzar el $20 \%$ del consumo energético de la UE. Por último se decide reducir el consumo energético primario en un $20 \%$ a través de mejoras en la eficiencia energética.

En 2008 se adoptó el paquete de energía y cambio climático que entró en vigor en junio del año siguiente. Las características básicas de este paquete son la modificación del sistema europeo de derechos de emisión, que pasa a ser menos generoso con la asignación de derechos, reduciendo gradualmente las asignaciones hasta obtener una reducción del $21 \%$ en 2020 en relación a los derechos otorgados en 2005, y aumentando la cantidad de derechos subastados. Se establecen además objetivos para los sectores no incluidos en el mercado europeo de emisiones en función de las posibilidades de los Estados miembros de tal forma que, en conjunto, se reduzcan las emisiones provenientes del sector del transporte, los usos residenciales, 
el sector agrícola y los residuos en un $10 \%$ en 2020 en relación a las emisiones de 2005. Se acuerda también repartir entre los Estados miembros la aportación de las renovables al mix energético para alcanzar el objetivo de lograr que un $20 \%$ del consumo energético provenga de energías renovables. Por último, se desarrolla el marco regulatorio para la captura y el almacenamiento de $\mathrm{CO}_{2}$ que apoya la puesta en marcha de plantas piloto hasta 2015 con objeto de disponer de la tecnología de captura y almacenamiento de carbono a escala comercial en $2020^{62}$.

Los tres últimos años de la sexta y última etapa en la política climática europea han estado marcados, entre otros acontecimientos, por las cumbres climáticas internacionales. En la Cumbre de Copenhague en 2009 la UE reconoció no haber cumplido con sus objetivos de alcanzar un acuerdo ambicioso, justo y legalmente vinculante que entrara en vigor en 2012. No obstante, el acuerdo de Copenhague ${ }^{63}$ contiene los elementos básicos de los Acuerdos de Cancún, que fueron aclamados como un éxito relativo por la práctica totalidad de la comunidad internacional, exceptuando Bolivia. En relación al papel de la UE durante la CdP15, la sensación fue de pérdida de liderazgo ${ }^{64}$ a favor de los grandes contaminadores (China, EEUU, India, Sudáfrica y Brasil).

En 2010 se celebró la CdP16 en Cancún. Tanto la comunidad internacional como la UE esperaban logros más modestos que los esperados para la CdP15. El resultado de Cancún ha sido valorado como un éxito relativo ya que ha habido una vuelta al multilateralismo, se han adoptado los Acuerdos de Cancún en el seno de la CMNUCC y se ha avanzado en temas operativos. Durante la CdP16 la labor de la UE ha sido reseñable por haber facilitado el acercamiento de posturas y haber ahondado en aspectos de gran relevancia para los países en desarrollo, como por ejemplo, en materia de adaptación en el ámbito internacional.

En 2011 Europa ha trazado una hoja de ruta para mediados de siglo que va a suponer la eliminación de las emisiones de $\mathrm{CO}_{2}$ casi por completo del sector eléctrico, así como reducciones significativas de los sectores difusos y de la industria. El gráfico 2, presentado a continuación, muestra en el eje de abscisas los años, y el eje de ordenadas muestra la contribución, expre-

${ }^{62}$ Para ver un análisis favorable al desarrollo de los mecanismos de captura y almacenamiento de carbono consúltese ÁLVAREZ PELEGRY, E., «La captura y almacenamiento de $\mathrm{CO}_{2}$ : una solución eficiente para luchar contra el cambio climático». Real Instituto Elcano. DT 20/2010-28/06/2010, 2010.

63 Para un análisis sucinto de los resultados de la Cumbre de Copenhague véase por ejemplo LÁZARO TOUZA, L., «Climate Change Talks: Breakdown in Copenhagen; Next Stop, Mexico 2010 (COP 16)», Real Instituto Elcano, ARI 9/2010-9/3/2010, 2010.

${ }^{64}$ Vid. JORDAN, A., et al., op. cit., nota 4, p. 3. 
sada en términos porcentuales, de los principales sectores a las emisiones de GEI. La línea roja muestra la tendencia con las políticas actuales.

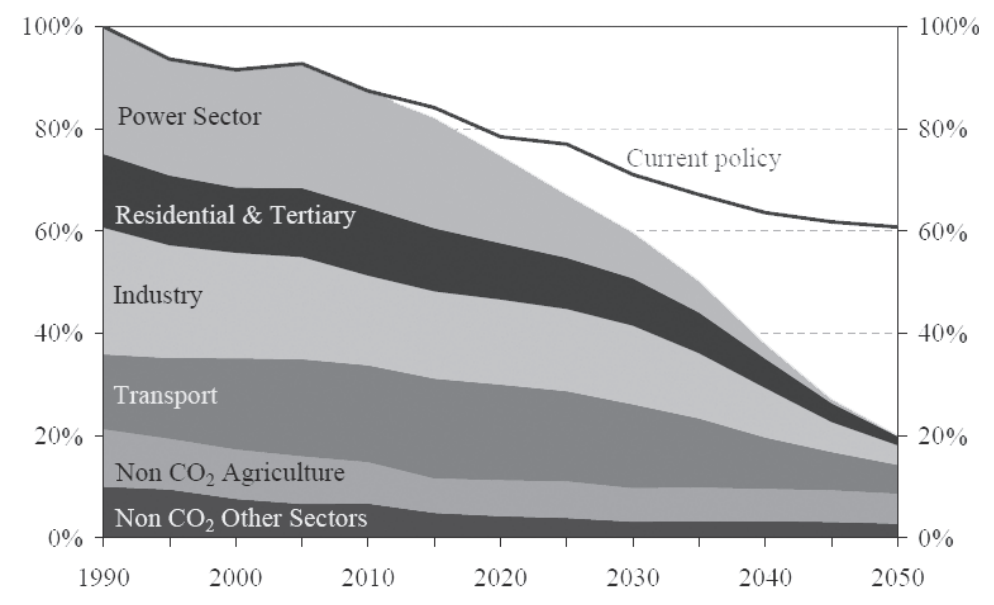

Fuente: EU COM (2011: 5) 112 final.

\section{Gráfico 2}

Emisiones de GEI hacia un 80\% de reducción en las emisiones (100\%=1990)

Según indica el Roadmap 2050 de la Comisión Europea, la transición hacia una economía menos intensiva en emisiones de GEI y competitiva supondrá reducir las emisiones en un $80 \%$ a mediados de siglo en relación a las emisiones de 1990. El análisis de la UE indica que una reducción de las emisiones del $40 \%$ en 2030 y del $60 \%$ en 2040 serían eficientes en términos de costes. Este esfuerzo supondría una reducción en las emisiones de GEI del $1 \%$ anual hasta 2020 , del $1.5 \%$ hasta 2030 y del $2 \%$ desde 2030 hasta 2050. Quedan, sin embargo, grandes retos por afrontar. El primer reto es cumplir con los compromisos de cada país. Pero esto no será suficiente como se verá a continuación.

\section{2. ¿Qué se ha conseguido hasta la fecha?}

Tal y como muestra el gráfico 3, los compromisos existentes en lo relativo a la mitigación sólo suponen el $60 \%$ de los compromisos necesarios para mantener el aumento de las temperaturas de $2^{\circ} \mathrm{C}$ en relación a la era preindustrial. En el eje de ordenadas podemos ver los años y en el eje de abscisas las emisiones globales totales medidas en giga toneladas de 
$\mathrm{CO}_{2}$ equivalente al año. El recuadro vertical nos muestra el rango en los compromisos adquiridos y el recuadro negro dentro del rectángulo nos indica la mediana en los rangos de compromisos. Como se puede observar $\operatorname{los} 2^{\circ} \mathrm{C}$ quedan aún lejos de los compromisos adquiridos hasta la fecha. Para situarnos dentro del rango de aumento de temperaturas recomendadas por la ciencia es necesario continuar haciendo esfuerzos a medio y a largo plazo.

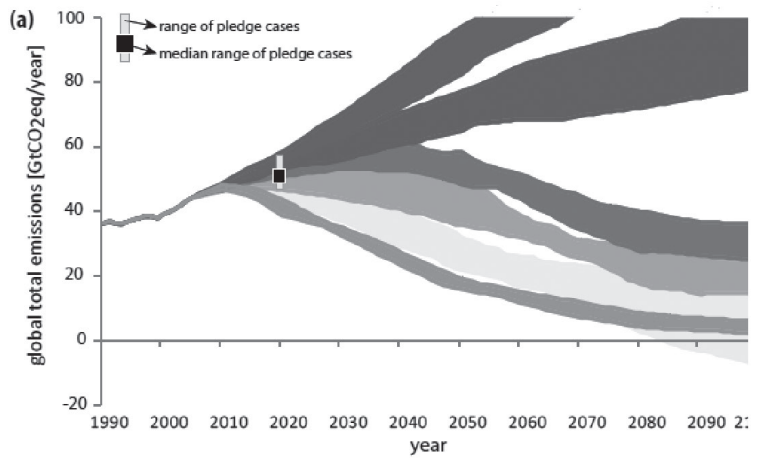

\section{Legend:}

Emission levels consistent with a likely temperature increase $(\mathrm{T})$ in the 21st century of:

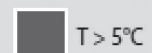

$\mathrm{T}>5^{\circ} \mathrm{C}$

$4^{\circ} \mathrm{C}<\mathrm{T}<5^{\circ} \mathrm{C}$

$3^{\circ} \mathrm{C}<\mathrm{T}<4^{\circ} \mathrm{C}$

$2.5^{\circ} \mathrm{C}<\mathrm{T}<3^{\circ} \mathrm{C}$

$2^{\circ} \mathrm{C}<\mathrm{T}<2.5^{\circ} \mathrm{C}$

$\mathrm{T}<2^{\circ} \mathrm{C}$

Fuente: UNEP (2010: 15).

\section{Gráfico 3}

Compromisos adquiridos y aumentos esperados de las temperaturas.

En relación a la financiación conseguida hasta la fecha, las cifras recopiladas por el World Resources Institute ${ }^{65}$ indican, en la última actualización de mayo de 2011, que en materia de financiación a corto plazo (fast start finance) se han conseguido compromisos de financiación de 28.144 millones de dólares (de los 30.000 millones de dólares aproximadamente que se habían acordado ${ }^{66}$. En principio, esta financiación de los países desarrollados a los países en desarrollo tiene que ser nueva y adicional a la ayuda oficial al desarrollo (AOD) y a otras ayudas comprometidas con anterioridad. Sin embargo, está todavía por ver si en realidad los fondos comprometidos

65 WORLD RESOURCES INSTITUTE, «Summary of Climate Finance Pledges Put Forward by Developed Countries», 2011.

66 Para ver las comunicaciones completas de finales de mayo de 2011 sobre financiación a corto plazo consúltese: http://unfccc.int/cooperation_support/financial_mechanism/fast_ start_finance/items/5646.php 
van a ser realmente nuevos y adicionales. Quedan por definir la procedencia y la gestión de la ayuda post 2013 según el compromiso adquirido en la CdP15 y en la CdP16 de los 100.000 millones de dólares anuales a partir de 2020. Sabemos que las arcas públicas en principio no podrán asegurar este volumen de financiación por si solas, por lo que se está trabajando en la obtención de dichos fondos.

En el ámbito de la adaptación al cambio climático se trabajó con especial énfasis desde España en la CdP16. Se estableció un marco para la adaptación así como un comité para la misma. Además se formularon un buen número de recomendaciones que incluyen el desarrollo de estudios de riesgos y de vulnerabilidad, la priorización de las acciones de adaptación y la necesidad de mejorar la capacidad de adaptación de los países más vulnerables, entre otros ${ }^{67}$.

Otro de los elementos clave de las negociaciones internacionales del clima es la verificación en las emisiones de GEI. Uno de los elementos que facilitan los acuerdos ambientales internacionales es la cesión limitada en la soberanía nacional. En este sentido, tras la CdP16 los países desarrollados deben presentar, adicionalmente a los informes anuales, un informe bianual sobre mitigación, adaptación, ayuda para la mejora de las capacidades de los países en desarrollo y ayuda en materia de transferencia tecnológica. Los países en desarrollo, por otra parte, deben someter al sistema de control internacional $\left(\mathrm{MRV}^{68}\right)$ las acciones en materia de lucha contra el cambio climático financiadas por los países desarrollados. Las acciones nacionales de los países en desarrollo no financiadas por los países desarrollados serán verificadas por un sistema internacional de consulta y análisis $\left(\mathrm{ICA}^{69}\right)$, respetando la soberanía nacional, petición hecha de manera reiterada por China.

En lo relativo a las transferencias de tecnología, los avances más importantes en la CdP16 fueron la creación del Mecanismo Tecnológico y de la Red de Tecnología Climática. El cometido principal de estas instituciones es el análisis de las necesidades tecnológicas y la elaboración de recomendaciones para la difusión de nuevas tecnologías.

Por último, dado que la deforestación causa hasta el 20\% de las emisiones de GEI, es interesante señalar los avances en esta área. Al finalizar la

${ }^{67}$ MMA, «Nota sobre los resultados de las negociaciones internacionales, tras la celebración de la 16. ${ }^{\text {a }}$ conferencia de las partes de la Convención Marco de Naciones Unidas sobre Cambio Climático y la $6 .{ }^{a}$ sesión de la conferencia de las partes que son partes del Protocolo de Kioto», 2010.

${ }^{68} \mathrm{MRV}$ es el acrónimo de monitorización, información y verificación (monitoring, reporting and verification).

${ }^{69}$ ICA es el acrónimo de International Consultation and Analysis. 
CdP16, el acuerdo de REDD-Plus ${ }^{70}$ quedaba formalizado ${ }^{71}$. En líneas generales se daba un enfoque gradual para que los países en desarrollo empezaran a tomar medidas para frenar la deforestación. Así se pedía la elaboración de inventarios, de planes nacionales para frenar la deforestación y la degradación forestal y se animaba a la inclusión de la población indígena y de las poblaciones locales en la gestión de los bosques. A pesar de que en 2011 las negociaciones están siendo más lentas de lo deseable, tardando días en llegar a acuerdos sobre, por ejemplo las agendas de negociación, se espera que haya avances en Durban relativos a la financiación de REDD-Plus.

\section{Retos pendientes}

A nivel global es fundamental que se cumpla con los compromisos adquiridos. Aunque la crisis económica ha afectado a todos los sectores, en la UE los recortes han afectado en menor medida a las políticas climáticas ${ }^{72}$. Una de las acciones recomendadas es aprovechar las oportunidades que ofrecen las medidas de eficiencia energética en el sector de la construcción, la automoción y la gestión de residuos sólidos urbanos. Dichas medidas, aun sin ser las más atractivas en términos de votos y aun dependiendo de la disponibilidad de financiación, podrían ayudar al cumplimiento de nuestros compromisos. Habrá no obstante que poner medidas para que el efecto «rebote» o rebound effect ${ }^{73}$ se limite.

Aun cumpliendo con los compromisos actuales estamos lejos de lograr evitar una interferencia peligrosa con el sistema climático, especialmente grave para los pequeños estados insulares y para zonas con estrés hídrico. Para lograr evitar una interferencia peligrosa con el clima es necesario aumentar el grado de compromiso en materia de mitigación de adaptación. Además, queda por determinar la procedencia exacta y la gestión de las transferencias de fondos.

Queda pendiente la difícil tarea de diseñar el futuro post 2012 que internalice el coste de las emisiones de $\mathrm{CO}_{2}$, haciendo uso de los mecanismos

70 REDD-Plus es un mecanismo negociado en el seno de la CMNUCC para reducir las emisiones (de GEI) provenientes de la deforestación, la degradación forestal y promueve la conservación, la gestión sostenible de los bosques y la mejora de los stocks de $\mathrm{CO}_{2}$ en los países en desarrollo.

${ }^{71}$ GRUBB, M., «Cancun: the art of the possible». Climate policy. 11, 2011, pp. 847-850.

72 Vid. HOBLEY, A., ADAMS, D., (2011), op. cit., nota 55, p. 20.

${ }^{73} \mathrm{El}$ efecto rebote (o rebound effect) es un concepto introducido en la literatura en relación con la eficiencia energética por Stanley Jevons en 1865 que hace referencia al aumento en la demanda debido a la reducción de costes derivados de aumentos en la eficiencia energética. 
de mercado y de mecanismos regulatorios de manera coordinada, para dar señales claras a empresas, gobiernos y ciudadanos. Parece claro que en el futuro habrá acuerdos parciales, con avances operativos en temas concretos y con mayor protagonismo de los países, las regiones y las ciudades ${ }^{74}$. No se espera a corto plazo un acuerdo ambicioso, justo y legalmente vinculante que incluya a todos los países por las razones expuestas a lo largo de las secciones anteriores, es decir, no hay incentivos suficientes para evitar el free-riding y para asegurar el cumplimiento de los compromisos adquiridos.

Un tema pendiente y de gran preocupación es la agricultura. Christiana Figueres admitía a mediados de febrero de 2011 que la discusión de cómo se va a alimentar a un planeta de 9.000 millones de habitantes a mediados de siglo, en un contexto climático previsiblemente más adverso, todavía no se había abordado con profundidad. Este es otro de los déficits de los acuerdos de Cancún.

La mitigación previsiblemente va a requerir una transformación sin precedentes de la matriz energética global. Hay regiones, como por ejemplo América Latina, que asisten a un proceso de «fosilización» en su mix energético y que podrían beneficiarse de un mayor uso de las energías renovables al tiempo que protegen el patrimonio forestal en forma de sumideros.

Por último, y sin duda clave en el desarrollo futuro de las negociaciones climáticas, tenemos el reto de sumar de manera responsable y decidida a EEUU. La discusión (inexistente en círculos académicos y en la literatura evaluada por pares) de la existencia de cambio antropogénico del clima sigue sorprendentemente viva en ciertos estados y en ciertos ámbitos en EEUU. La génesis de este país, con 13 estados iniciales que lograron imprimir un carácter de gran independencia y poder a los estados, junto con la ausencia de la disciplina de partido, sumado todo ello a una captura regulatoria (derivada de la financiación de los candidatos locales, regionales y federales) hace que sea extremadamente difícil adoptar en la actualidad una política climática decidida en EEUU, lo cual dificulta la cooperación internacional. Barack Obama invirtió una buena parte de su capital político en la reforma sanitaria y al llegar la hora de negociar la legislación sobre cambio climático, las rencillas históricas en este ámbito entre demócratas y republicanos (por haber abanderado Al Gore la lucha contra el cambio climático, entre otros elementos) han bloqueado cualquier avance en este tema. La única forma de convencer a ciudadanos, empresas y gobiernos de distinto signo político en EEUU de la necesidad de reducir las emisiones de GEI es

${ }^{74}$ La relevancia de las ciudades se deriva del hecho de que emiten entre el $60 \%$ y el $80 \%$ de las emisiones globales de GEI y consumen aproximadamente esas mismas cantidades de energía. Véase OECD, Cities and Climate Change. París: OECD Publishing, 2010. 
hablar de ahorro energético, hablar de aumento de la independencia energética a través de reducciones de las importaciones de combustibles de zonas inestables, hablar de la creación de empleos de «cuello verde» (green collar jobs) y de innovación ${ }^{75}$. El reto pendiente por tanto es disponer de la información económica sobre costes y beneficios de la acción climática, difundirla y lograr el compromiso de EEUU en la reducción de GEI.

\section{Conclusiones}

Sabemos, como decía Christiana Figueres, que el océano no se cruza con un sólo soplo de viento. En este sentido, desde los ciudadanos y las ciudades hasta los grandes contaminadores históricos (UE o EEUU) y futuros (China y otros países no incluidos en el anexo I del Protocolo de Kioto) están llamados a cooperar, aunque sea de manera gradual y por sectores, si queremos cumplir con el objetivo de no interferir de manera peligrosa con el clima. Los elementos facilitadores de los acuerdos ambientales internacionales vistos con anterioridad indican la dificultad de llegar a acuerdos ambiciosos. La cooperación es por tanto necesaria pero no inevitable.

Dado el peso que tiene la energía en las emisiones de GEI el desarrollo del sector energético va a ser crucial para cumplir con los objetivos de mitigación. Empezando por las medidas de ahorro y eficiencia energética, que pueden suponer beneficios marginales netos, y, continuando por la innovación y una mayor penetración de las energías renovables, el camino está marcado por una transformación sin precedentes de cómo valoramos y cómo usamos la energía. En el ámbito de la adaptación se ha resaltado en el artículo que el agua será la gran protagonista. Por lo tanto, el nexo energíaagua será un área interesante de investigación en el futuro ${ }^{76}$.

En lo relativo al papel que ha jugado históricamente la UE en la gobernanza global del cambio climático, se ha argumentado que, a pesar de la complejidad y, en ocasiones, ambigüedad en el uso del término liderazgo, parece razonable afirmar que la UE ha ejercido un liderazgo direccional marcado por el ejemplo dado, los sacrificios hechos y los valores defendi-

${ }^{75}$ La autora agradece el apoyo del Departamento de Estado del Gobierno de EEUU y de su programa de becas Fulbright (Programa de energías renovables y cambio climático dentro de la iniciativa International Visitor Leadership Program) que le permitió conocer de cerca las políticas y los procesos de toma de decisiones en los procesos de gobernanza del cambio climático en EEUU.

${ }^{76}$ LÁZARO TOUZA, L., LÓPEZ-GUNN, E., «Climate change policies: mitigation and adaptation at the local level. The case of the city of Madrid (Spain)» en TORTORA, M., (Coord.) (en imprenta). Sustainable Systems and Energy Management at the Regional Level: Comparative Approaches, IGI Global, en imprenta. 
dos. Como se ha argumentado, la influencia de un líder depende de varios factores: el potencial para guiar, la motivación y las capacidades. En este sentido se han analizado los elementos del liderazgo europeo así como los momentos clave en los que la UE ha liderado el proceso de gobernanza global del clima. El análisis muestra una evolución desde la práctica inexistencia de políticas climáticas al desarrollo de una suma de políticas individuales, para llegar en los últimos años del siglo XX y principios del XXI a una política climática europea.

En relación a la influencia y al liderazgo direccional, la UE, entre otras iniciativas, abogó en las negociaciones internacionales por un enfoque de objetivos vinculantes de reducciones de GEI y tiempos para cumplirlos, fortaleció la CMNUCC, «salvó» el proceso negociador cuando EEUU decidió no mandar al senado el Protocolo de Kioto para su ratificación, aceptó las reducciones de GEI más ambiciosas del Protocolo de Kioto, negoció con Rusia para la ratificación del PK en 2004, puso en marcha el primer mercado transnacional de derechos de emisión, acordó reducciones unilaterales de emisiones del $20 \%$ en 2020 y ha presentado una hoja de ruta para «descarbonizar» la economía a mediados de siglo. A medida que los objetivos de reducción sean más elevados y más costosos, las divergencias en el seno de la UE, entre líderes y rezagados, y fuera de ella, entre países desarrollados y países en desarrollo, previsiblemente complicarán las negociaciones climáticas internacionales, especialmente en contextos económicos adversos.

Desde los inicios de la preocupación europea por el clima hasta la actualidad se han dado varias etapas, más o menos exitosas en lo relativo a negociaciones internas e internacionales. Sin embargo, tanto por las consecuencias esperadas del cambio climático para la UE como por las oportunidades económicas, apoyo de ciudadanos y apoyo de Estados miembros como Alemania o el Reino Unido, entre otros, la UE sigue avanzando para, como decía el Presidente de la Comisión Europea José Manuel Durao Barroso, «abrazar nuestro futuro bajo en carbono».

\section{Bibliografía}

ALCARÁZ VARÓ, E., HUGHES, B., Diccionario de términos económicos, financieros y comerciales. Barcelona, Arial Referencia, 2002.

ÁLVAREZ PELEGRY, E., «La captura y almacenamiento de $\mathrm{CO}^{2}$ : una solución eficiente para luchar contra el cambio climático», Real Instituto Elcano. DT 20/2010-28/06/2010, 2010.

ANDERSEN, S., AGRAWALA, S., «Leaders, Pushers and Laggards in the Making of the Climate Regime», Global Environmental Change, 12, 2002, pp. 41-51.

ASSADOURIAN, E., «Cultural Change for a Bearable Climate», Real Instituto Elcano. ARI 163/2010-17/11/2010, 2010. 
BARRETT, S., Why Cooperate? The Incentive to Supply Global Public Goods, Oxford University Press, Nueva York, 2007.

BARRETT, S., «On Theory and Diplomacy of Environmental Treaty-making», Environmental and Resource Economics. 11(3-4), 1998, pp. 317-333.

BRETTEVILLE-FROYN, C., «International Environmental Cooperation: The Role of Political Feasibility», en Atkinson, G., et al., (eds.), Handbook of Sustainable Development, Cheltenham, Edward Elgar, 2007.

BOSETTI, V., DECIAN, E., «A gook opening. The key to make the most of multilateral action». Fondazione Eni Enrico Mattei. Documento de Trabajo presentado en EAERE 2011, Roma, 29 de junio-2 de julio de 2011.

BYATT, I., et al., «The Stern Review "OXONIA Papers": A Critique», World Economics. 7(2), 2006, pp. 145-150.

CARTER, et al., «The Stern Review: A Dual Critique», World Economics, 7(4), 2006, pp. 165-232.

COM (2011) 370 final. Proposal for a Directive of the European Parliament and of the Council on energy efficiency and repealing Directives 2004/8/EC and 2006/32/EC, 22 de junio de 2011.

COM (2011) 112 final. «A Roadmap for moving to a competitive low carbon economy in 2050», 8 de marzo de 2011.

COM (2011) 21, «A resource-efficient Europe - Flagship initiative under the Europe 2020 Strategy», 26 de enero de 2011.

COM(2010) 265 final, Comunicación de la Comisión al Parlamento Europeo, al Consejo, al Comité Económico y Social europeo y al Comité de las Regiones, Análisis de las opciones para rebasar el objetivo del $20 \%$ de reducción de las emisiones, de gases de efecto invernadero y evaluación del riesgo de fugas de carbono, 26 de mayo de 2010.

COM (2009) 147 final, White Paper. Adapting to climate change: towards a European framework for action, 1 de abril de 2009.

DELLINK, D., et al., «Sharing the burden of financing adaptation to climate change». Global Environmental Change. 19, 2009, pp. 411-421.

DIETZ, S., et al., «Reflections on the Stern Review (1), A Robust Case for Strong Action to Reduce the Risks of Climate Change», World Economics, vol. 8, n. ${ }^{\circ}$, 2007, pp. 121-168.

European Environment Agency, (EEA), Annual EC greenhouse gas inventory 19902006 and Inventory Report, EEA Technical Report 6/2008, Copenhagen, European Environment Agency, 2008.

ELOLA, T., «La representación de la UE en las negociaciones internacionales sobre el cambio climático tras la entrada en vigor del Tratado de Lisboa», Real Instituto Elcano, ARI 97/2011-25/05/2011, 2011.

FANKHAUSER, S., HEPBURN, C., PARK, J., «Combining multiple climate policy instruments: how not to do it», Climate Change Economics, Vol. 1, n. ${ }^{\circ}$, 2010, pp. 1-17.

FISHBEIN, M., AJZEN, I., 'Belief, Attitude, Intention and Behaviour: An Introduction to Theory and Research. Reading, MA, Addison -Wesley, 1975.

FMI, Economías en transición: Análisis del FMI sobre los avances y las perspectivas. Noviembre de 2000, 2000. 
GERHARDS, J., LENGFELD, H., «"The growing remit of the EU in environmental and climate change policy and citizens" support across the Union», European Journal of Social Policy, 2008.

GILKSON, A., «Climate Change. A Response to "The Stern Review": A Dual Critique», World Economics, vol. 8, n. ${ }^{\circ}$ 1, 2007, pp. 233-238.

GRUBB, M., «Cancun: the art of the possible», Climate policy, 11, 2011, pp. 847-850.

HOBLEY, A., ADAMS, D., «The EU and Climate Change in the lead up to Cancun: Impacts, policies and Positions», Real Instituto Elcano, ARI 166/210. 24/11/2010, 2010.

HUITEMA, D. et al., «The evaluation of climate policy: theory and emerging practice in Europe», Policy Sciences, DOI 10.1007/s11077-011-9125-7, 2011.

Intergovernmental Panel on Climate Change (IPCC), Fact sheet: Climate change science - the status of climate change science today, 2011.

IPCC, Climate Change 2007, Synthesis Report, 2007.

JAEGER, C., et al., A New Growth Path for Europe. Generating Prosperity and Jobs in the Low-Carbon Economy. Synthesis Report. European Climate Forum, 2011.

JORDAN, A., et al., Climate Change Policy in the European Union. Confronting the Dilemmas of Mitigation and Adaptation, Cambridge, Cambridge University Press, 2010.

KOETZ, T., et al., «Building better science-policy interfaces for international environmental governance: assessing potential within the Intergovernmental Platform for Biodiversity and Ecosystem Services», International environmental Agreements, DOI 10.1007/s10784-011-9152-z, 2011.

LÁZARO TOUZA, L., LÓPEZ-GUNN, E., «Climate change policies: mitigation and adaptation at the local level. The case of the city of Madrid (Spain)» en TORTORA, M., (coord.), Sustainable Systems and Energy Management at the Regional Level: Comparative Approaches, IGI Global, en imprenta.

LÁZARO TOUZA, L., «Cancún: anclando Copenhague y salvando el proceso para salvar el clima... quizá mañana». Real Instituto Elcano, ARI 12/201120/01/2011, 2011.

LÁZARO TOUZA, L., «Climate Change Talks: Breakdown in Copenhagen; Next Stop, Mexico 2010 (COP 16)», Real Instituto Elcano, ARI 9/2010-9/3/2010, 2010.

LÁZARO TOUZA, L., «Cambio climático: ¿Combinación de instrumentos para "Un post-Kioto feliz?"», Real Instituto Elcano, ARI N. ${ }^{\circ}$ 12/2008, 2008.

MARÍN GONZÁLEZ, M., «Seguridad, Modelo Energético y Cambio Climático» en MINISTERIO DE DEFENSA (ed.), Seguridad, Modelo Energético y Cambio Climático. Cuadernos de Estrategia, Instituto Español de Estudios Estratégicos, N. ${ }^{\circ} 150$, enero, 2011, pp. 15-25.

MMA, «Nota sobre los resultados de las negociaciones internacionales, tras la celebración de la 16. ${ }^{a}$ conferencia de las partes de la Convención Marco de Naciones Unidas sobre Cambio Climático y la 6. ${ }^{\mathrm{a}}$ sesión de la conferencia de las partes que son partes del Protocolo de Kioto», 2010.

NEUMAYER, E., Greening Trade and Investment. Environmental Protection Without Protectionism, London, Earthscan, 2001. 
NEUMAYER, E., «In Defence of Historical Accountability for Greenhouse Gas Emissions», Ecological Economics, 33 (2), 2000, pp. 185-192.

NORDHAUS, W. D., "A Review of the Stern Review on the Economics of Climate Change», Journal of Economic Literature, Vol. XLV, 2007, pp. 686-702.

OBERTÜR, S., «Global Climate Governance after Cancun: options for EU leadership». The International Spectator. Vol. 46, 2011, pp. 5-13.

ORBERTHÜR, S., ROCHE KELLY, C., «EU Leadership in International Climate Policy. Achievements and Challenges». The International Spectator. Vol. 43, 2008, pp. 35-50.

OECD, Cities and Climate Change. París, OECD Publishing, 2010.

PÉREZ DE LAS HERAS, B., (coord.), The European Union Greenhouse gas Emissions Trading Scheme: Abilities and Prospects of a Climate Governance Instrument. Navarra: Editorial Aranzadi y Thomson Reuters, 2010.

PERKINS, R., «Environmental leapfrogging in developing countries: A critical Assessment and Reconstruction». Natural resources Forum. 27, 2003, pp. 177-188.

PEZZEY, J., «Sustainability: An Interdisciplinary Guide». Environmental Values, 1(4) 1992, pp. 321-362.

SANDLER, T., «Protocolo de Kioto: dificultades para la acción colectiva», Papeles de economía española. Economía y Cambio Climático, n. ${ }^{\circ}$ 121, 2009, pp. 14-24.

SKOVDIN, T., ANDERSEN, S., «Leadership Revisited». Global Environmental Politics, Volume 6, Number 3, 2006, pp. 13-27.

STERN, N., et al., The Economics of Climate Change. The Stern Review, Cambridge, Cambridge University Press, 2006.

STERN, N., «Managing Climate Change and Overcoming Poverty: Facing the Realities and Building a Global Agreement», London, London School of Economics, 2009.

UNDERAL, A., «Solving Collective Problems: Notes on Three Modes of Leadership» en Challenges of a Changing World: Festchrift to Willy Østreng, 139-153. Lysaker, Norway: The Fridtjof Nansen Institute, 1991.

UNEP, Green Jobs Report. Some Facts and Figures and Highlights, 2008.

UNEP, Emissions Gap Report. Are the Copenhagen Accord Pledges Sufficient to Limit Global Warming to $2^{\circ} \mathrm{C}$ or $1.5^{\circ} \mathrm{C}$ ? A preliminary assessment. November 2010, 2010.

UNITED STATES DEPARTMENT OF ENERGY (US DoE), 2010 Buildings Energy Data Book. Energy Efficiency and Renewable Energy. March 2011, 2011.

WORLD RESOURCES INSTITUTE, «Summary of Climate Finance Pledges Put Forward by Developed Countries», 2011.

WORLD WIND ENERGY ASSOCIATION, World Wind Energy Report 2010, 2010. 